Article

\title{
Design of Explosives Detector Sensors Based on GFETs
}

\author{
Saeid Masoumi 1,* Hassan Hajghassem ${ }^{2}$, Alireza Erfanian ${ }^{3}$ and Ahmad Molaei Rad ${ }^{4}$ \\ 1 Malek Ashtar University of Technology, Department of Electronic Engineering; s.masoumi.ee@gmail.com \\ 2 Tehran University, Department: Faculty of New Sciences \& Technologies; hajghassem@ut.ac.ir \\ 3 Malek Ashtar University of Technology, Department of Electronic Engineering; Erfanian@mut.ac.ir \\ 4 Malek Ashtar University of Technology, Department of Bioscience and Biotechnology; Molaeirad@gmail.com \\ * Correspondence: s.masoumi.ee@gmail.com; Tel.: +98-914-471-1362
}

\begin{abstract}
Smart sensors based on graphene field effect transistor and biologically receptors are regarded as a promising nanomaterial that could be the basis for future generations of selective real-time monitoring of target analytes and smaller electronics. So the purpose of this paper is to provide details a real-time and selective explosive sensor based on GFETs and PDA-based lipid membranes coupled with biologically inspired TNT peptide receptors. Following an introduction, this paper describes the way of fabrication of the GFETs device by investigation methods for transferring graphene sheet from $\mathrm{Cu}$ substrates to target substrates, which is functionalized by the TNT peptide receptors, in order to offer a system which has the capability of answering the presence of related target molecules. Field effet transistor was fabricated using graphene as a channel and monitored by the source-drain current and back-gate voltage curves in the measurement. The transport property changed compared to that of the FET made by intrinsic graphene, that is, the Dirac point position moved from positive $\mathrm{Vg}$ to negative $\mathrm{Vg}$, indicating the transition of graphene from p-type to n-type after annealing in TNT, and GFET sensor show good sensitivity and selectivity response.
\end{abstract}

Keywords: GFETs; TNT explosive; sensor; TNT peptide receptor; graphene sheet

\section{Introduction}

The ability to detect and analyze volatile organic compounds (VOCs) such as explosives, pesticides, disease markers, and food aromas has significant bearing on our security, health, and general well being. The selective detection of a specific target molecule in a complex environment containing potential contaminants presents a significant challenge in biological sensor development. In 2000, Kong et al. first demonstrated a nanomaterials-based gas sensor [1]. Electronic materials with inherent nanoscale features are ideal components for next generation autonomous sensor technology by efficiently combining excellent detection sensitivity with ballistic charge transport in a single layer of material. During the past decades, nanomaterial-based FET biosensors, such as one-dimensional (1D) Si Nano-wire (SiNW) and carbon nanotube (CNT) devices have attracted significant attention for label-free detection of chemical and biological species. Recently, the highly sensitive and selective graphene FET biosensors have been developed in detecting biomolecules [2]. Graphene exhibits many fascinating physical properties, such as the extremely high carrier mobility 
and saturation velocity, making it very attractive in various applications, including transparent electrodes, transistors, sensors and so forth [3]. Graphene is an archetypal two-dimensional single atom thick material with sp2 bonded carbon atoms faultlessly arranged in a honeycomb lattice, and it is considered to be one of the most attractive materials [4]. Researchers have spared no effort to make great progress to further facilitate and improve the properties and to broaden the range of applications from this newly developed class of materials [4]. First of all, the advancements in graphene fabrication method, from the easiest mechanical cleavage to more complex and higher quality construction, are both enormous and remarkable despite such a short period of time [4]. However, its ambipolar behavior and Nano electronic conductance play an important role in the application of electronic devices, especially for graphene based field effect transistors. GFETs are widely applied in physical, chemical and biological sensing field [4]. Utilizing phage display techniques against trinitrotoluene (TNT) and dinitrotoluene (DNT) targets, peptide receptors have previously been identified with selective binding capabilities for these molecules. For practical applications, these receptors must be immobilized onto the surface of sensor platforms at high density while maintaining their ability to bind target molecules. In the case of explosive detection, highly selective coatings capable of discriminating between TNT, DNT, and other explosive molecules have been achieved through the use of molecule specific antibodies [5], [6]. TNT is one of the best known explosives, but it is also recognized as an environmental pollutant distributed in air, water, and soil. Due to its toxicity, TNT exposure can result in severe adverse effects on human health. In this timeframe of history, sensors based GFETs are required for different uses including clinical diagnosis technologies, environmental tests, and bioterrorism recognition technologies Corresponding to the military conflicts and terrorism. So, GFET sensor design provides real-time detection of TNT explosives. Therfore, first the field effect transistor with nanomaterials will be designed and created by graphene [7] and then biological receptor of TNT will be studied and fabricated, and then GFETs is functionalized.

\section{Graphene Based Field Effect Transistors}

Electric field effect on graphene was one of the outstanding results showed by Novoselov et al. [8]. As a consequence, extensive amount of research has been done on graphene field effect transistors (GFETs) in which graphene is used as the channel material. The high carrier mobility and unique band structure of graphene make it extremely useful for FETs application. So studies on more stable FETs with higher carrier mobility and better miniaturization potential have been investigated. FETs using graphene as the channel material have been developed to overcome the obstacles encountered by Si nanowires, CNTs, organic materials, and so on. Furthermore, graphene based FETs (GFETs) will continue to follow Moore's law to realize the further miniaturization of integrated circuits. Single layer graphene, a zero band gap semimetal, its Dirac fermion can be converted from electron to hole (or hole to electron) continuously under the electric field. While the electric field and the concentration of electric charge carriers are both zero, we call it a Dirac point [4]. The one fold charge carrier exists far from the Dirac point in graphene, its concentration and gate voltage can be described as ne, $\mathrm{b}=\alpha \mathrm{Vg}$. Here, ne is electric concentration, $\mathrm{nb}$ is hole concentration, $\alpha$ is charge injection rate in the gate, $\mathrm{Vg}$ is gate voltage. The resistance is given in Equation 1, hall coefficient is given in Equation 2. These carriers can change continuously between electric and holes by the increasing the ambipolar gate voltage. Positive gate voltage makes graphene an electron conductor while negative voltage make it a hole conductor. 


$$
\begin{aligned}
& \frac{1}{\rho}=e n \mu \\
& R_{h}=\frac{1}{e n}
\end{aligned}
$$

In experiments, resistance (or conductivity) and hall coefficient can prove that the gate voltage can effect carriers of graphene. The carriers transit from electrons (holes) to holes (electron) while approaching the Dirac point, and hall coefficient change its sign symbol (electron-positive and holenegative).

\subsection{Structures of GFETs}

The high carrier mobility and the unique band structure of graphene make it promising for diverse electronic applications, especially in FETs applications aspect. And next, the various structures of GFETs will be introduced.

\subsection{Back-gated GFETs}

Figure 1 (a) shows the general structure of GFETs and defines the main variables used in this paper. Thermodynamically stable graphene sheet was experimentally discovered in 2004 by Giem and Novoselov [8]. Novoselov's team fabricated the first back-gated GFETs with graphene films prepared by mechanical exfoliation of small mesas of highly oriented pyrolytic graphite [8]. In the beginning, back-gate devices were being fabricated from exfoliated graphene on $\mathrm{Si} / \mathrm{Sio} 2$ substrate in which silicon and silicon dioxide served as back gate electrode and dielectric respectively. However, since top-gate devices are much more desirable for applications, it did not take long for the first top-gate graphene field effect device to emerge in 2007 [9].

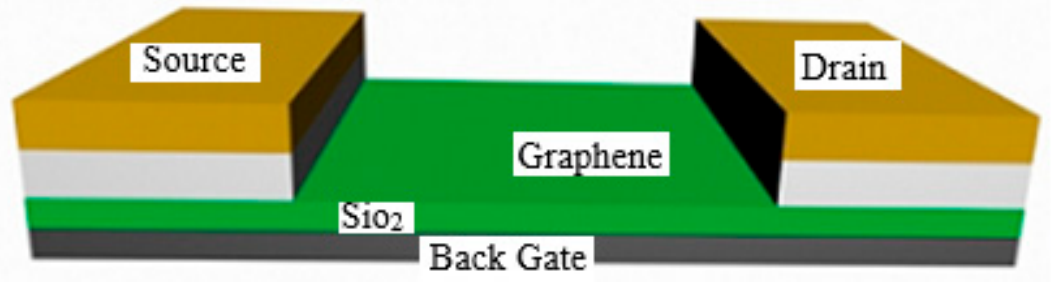

Figure 1. Back-gate GFETs, P Si wafer (as a gate electrode), Sio2, Graphene (as a channel), Cr \& Au as a source and darain electrodes.

The operation of am-bipolar graphene transistors differ significantly from unipolar $\mathrm{Si}$ MOSFETs. The channel charges contributing to current are always electrons in n-channel $\mathrm{Si}$ MOSFETs and always holes in p-channel Si MOSFETs in all regions of operation of the devices. In GFETs, however, conduction in this zero-band-gap material is by electrons at a high gate bias when the quasi-Fermi level (for electrons) is above the charge neutrality point at every point in the channel and by holes at low gate biases when the quasi-Fermi level (for holes) is below the charge neutrality point anywhere in the channel, as shown in Figure 2 (a), (b). However, in the region where the device channel transits from n-type to p-type, conduction is by both electrons and holes. This is called the ambipolar region (see region II in Figure 2). Assuming that VDS $>0$, channel conduction in this region is by electrons injected from the source and holes injected from the drain, which meet and recombine in pairs at a point in the channel. Hence, the channel conduction in GFETs exhibits characteristics of an n-type FET, a p-type FET, and also a transitional ambipolar behavior with both n-type and p-type sections in the channel, depending on bias conditions. The model proposed in this section divides the characteristics of GFETs into these three operation regions. The boundaries between the three operation regions are determined by the position of the recombination point (also 
called "the minimum charge point" in some literature) along the channel for given bias conditions. Specifically, the device is in the ambipolar region (i.e., region II) if the recombination point exists between the source and the drain in the channel. Otherwise, the device is in region I or III.

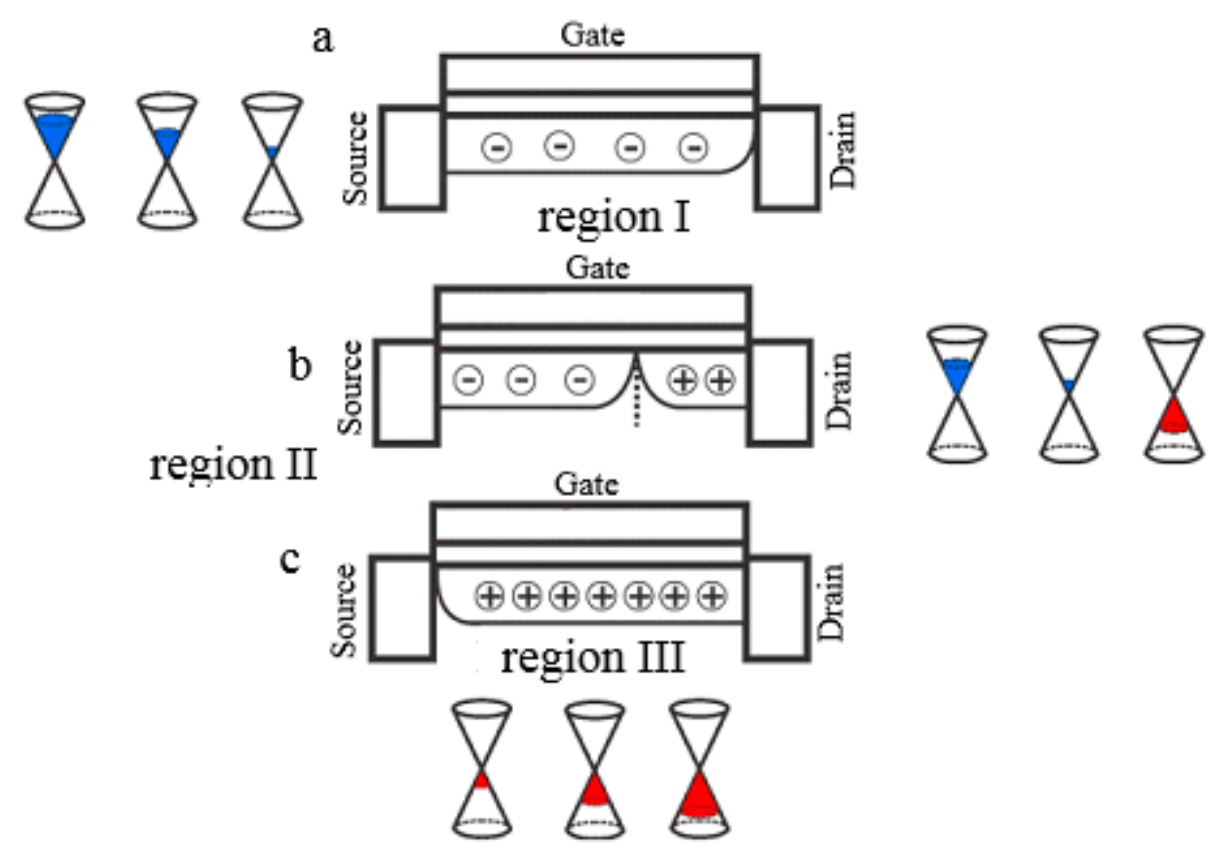

Figure 2. Conical-shaped band structure and distribution of charge carriers in the channel at different operation regions of GFETs. Assuming that VDS > 0, in region I, the device has n-type carriers everywhere in the channel. In region II, the device has n-type carriers at the source side of the channel and p-type carriers at the drain side of the channel. This is the ambipolar region. Point $\mathrm{X}$ is the recombination point. In region III, the device has p-type carriers everywhere in the channel.

\section{Fabricating transistor}

In this work, back-gate graphene field effect transistors were fabricated using graphene sheet on $\mathrm{Si} / \mathrm{Sio} 2$ substrate. Silicon dioxide film was also served as back gate dielectric.

\subsection{Substrate preparation}

In order to fabricate field effect transistor, p-type $<100>$ wafer with a thickness of $400 \mu \mathrm{m}$ available in the laboratory with $30 \mathrm{ohm} / \mathrm{Cm}$ resistance properties were used. Wafers are cut in $1.45 \times 1.45 \mathrm{Cm} 2$ dimensions. To clean the samples we use RCA method, 2 part $\mathrm{H} 2 \mathrm{o}, 2$ part $\mathrm{NH} 4 \mathrm{OH}$ and 2 part $\mathrm{H} 2 \mathrm{o} 2$ solution. Put the provided solution on heater with $80 \pm 5{ }^{\circ} \mathrm{C}$ temperature and submerge the samples in it for 15 minutes. Then, wash the samples in a deionized water container for 10 minutes at ambient temperature. And finally, expose samples to wind and clean them. An appropriate substrate requires an insulator film with specific thickness for identification of graphene on the substrate. Therefore, fabrication process started with thermal oxidation of p-type silicon wafer in oxidation furnace. In order to have maximum contrast in visible light, $300 \mathrm{~nm}$ oxide thicknesses was chosen and $345 \mathrm{~nm}$ practical thicknesses was measured by ellipsometry. Oxygen flow in the dry oxidation is set at about $2 \mathrm{Sccm}$. In addition, a set of e-beam alignment marks was needed for e-beam lithography steps. The utilized pattern is designed with marker mask for aligning 
in photolithography processes such as graphene sheets Patterning, deposition of electrodes as shown in Figure 3. The Cr electrodes (30 nm Cr) [10] with $\mathrm{W}=30 \mu \mathrm{m}, \mathrm{L}=56 \mu \mathrm{m}$ and $\mathrm{W}=60 \mu \mathrm{m}, \mathrm{L}=56$ $\mu \mathrm{m}$, were fabricated on Sio2 $(300 \mathrm{~nm}) / \mathrm{Si}$ substrate to make aligning markers using conventional macro-Nano processing technologies including photolithography, lift-off process and electron-beam evaporation. A large area between the electrodes markers due to locating to part of the graphene sheets created under the electrodes, in order to get a better connection between the source and drain electrodes.
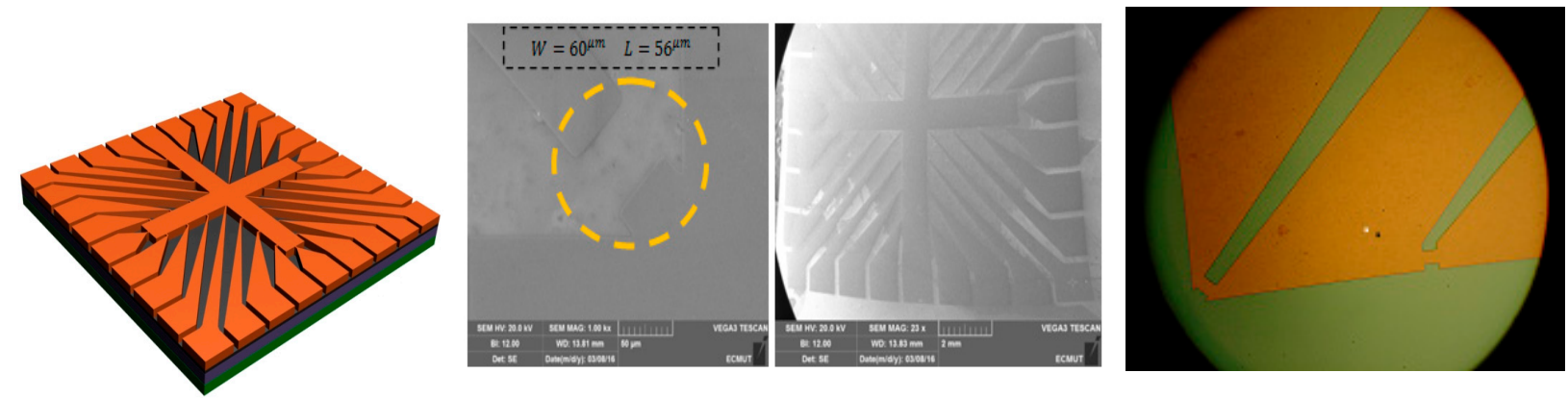

Figure 3. Markers mask (Si/Sio2/Cr: $30 \mathrm{~nm}$ ), SEM image of markers mask and optical microscopy images of the aligning markers electrodes.

\section{Graphene transfer process}

One common method for transferring graphene from a transition metal growth substrate is the "PMMA- mediated" approach [11], [12]. In this method a layer of poly(methyl-methacrylate) (PMMA) is coated onto the graphene, and the metal below it is etched away completely by etchant. The PMMA/graphene stack is then transferred onto another substrate, and solvents are used to remove the PMMA and complete the graphene transfer [13]. This approach is relatively simple, making it a popular transfer method [13]. Copper is commonly used as a substrate for CVD growth of graphene, while the popular chemical etchants for graphene transfer include aqueous solutions of iron nitrate [14], iron chloride [15] and ammonium persulfate. Monolayer Graphene on $\mathrm{Cu}(10 \mathrm{~mm} x$ $10 \mathrm{~mm}$ ) with thickness (theoretical) $0.345 \mathrm{~nm}$ is purchase from Graphenea of Spain. To transfer the graphene sheet from copper foil on the substrate, follow these steps to do so. Step1: Backside graphene etch By O2 plasma for $30 \mathrm{Sec}$ at a power of $100 \mathrm{~W}$. Preparation $4 \%$ solutions $(46 \mathrm{mg} / \mathrm{mL})$ of PMMA in anisole. Then PMMA spin-coating at $4500 \mathrm{rpm}$ for $1 \mathrm{~min}$ on a $1.45 \times 1.45 \mathrm{~cm} 2$ piece of graphene/Cu with thickness $300 \mathrm{~nm} \&$ bake at $180^{\circ} \mathrm{C}$ for $1 \mathrm{~min}$. Backside graphene etch by $\mathrm{O} 2$ plasma for $45 \mathrm{~S}$ at a power of $100 \mathrm{~W}$. The $\mathrm{Cu}$ substrate under the graphene film was etched in an iron nitrate solution with a concentration of about $0.7 \mathrm{~mol} / \mathrm{L}$ in water as shown in Figure 4.
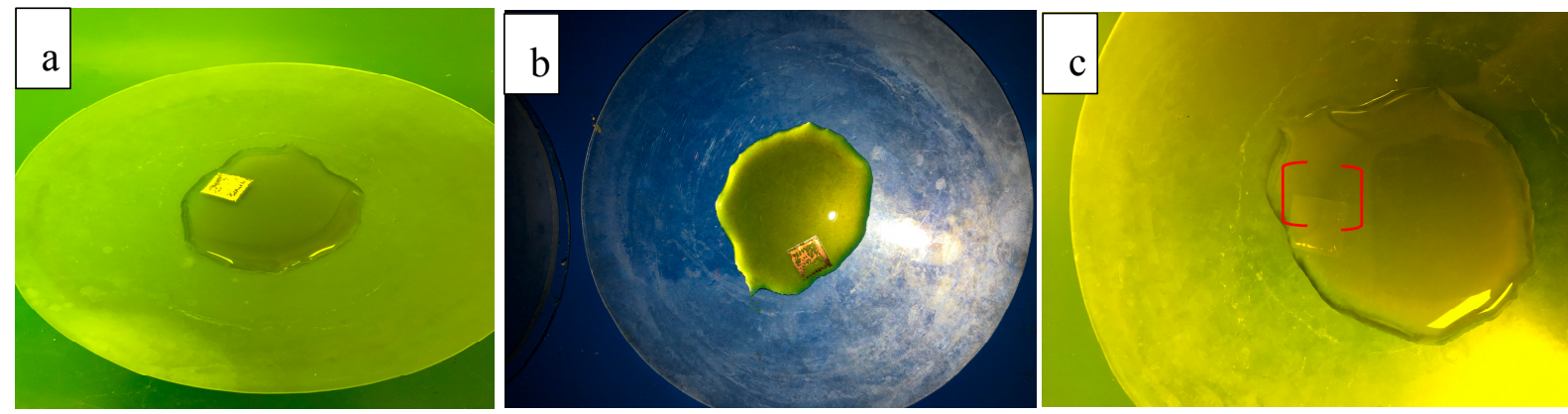

Figure 4. a) The etching of copper foil, b) after 50 minutes, c) after $4 \mathrm{~h}$, all copper foil is etched. 
After etching of the copper foil graphene/PMMA in distilled water for 10 minutes and rinse it. Then removes ionic and heavy metal atomic contaminants by using a solution of 25:1:1 $\mathrm{H} 2 \mathrm{O} / \mathrm{H} 2 \mathrm{O} 2 / \mathrm{HCl}$ and cleaning the residual metal particles remaining on the PMMA/graphene stack. The PMMA/graphene stack was rinsed by DI water. The next stage is intended for the removal of insoluble organic contaminants with a 25:1:1 H2O/H2O2/ $\mathrm{NH} 4 \mathrm{OH}$ solution. To avoid damaging the graphene/PMMA stack in the bubbles created by the $\mathrm{H} 2 \mathrm{O} 2$, process of washing done at room temperature. After each etching step, the PMMA/graphene stack was rinsed by DI water. To transfer the graphene/PMMA stack, the oxide substrate with piranha solution is activated. Piranha solution (750 micro liters of $\mathrm{H} 2 \mathrm{O} 2$ with 250 micro liters of sulfuric acid) increases its hydrophilicity due to an increase in the density of the $\mathrm{OH}$ groups on the Sio2 surface and improves the smoothness of the graphene/PMMA. The increase in the hydrophilicity significantly reduces the formation of large folds and wrinkles, and the PMMA/graphene stack can be transferred without large fold formation at all. Other techniques that can increase the hydrophilicity of the targeting substrate will have similar effects on reducing the folds and cracks. For example, oxygen plasma treatments also increase the hydrophilicity of Sio2 substrates [16], [17]. To dry the residual water thoroughly, the sample was baked at $150^{\circ} \mathrm{C}$ for $15 \mathrm{~min}$ to evaporate it and improve the contact between the stack and the substrate as shown in Figure 5.
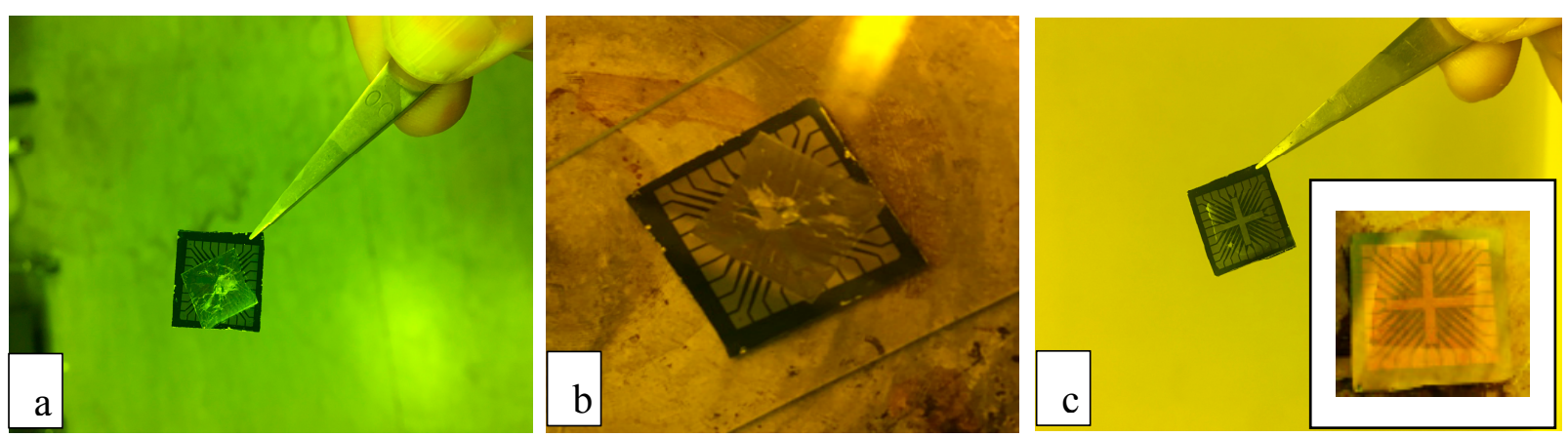

Figure 5. a) Transfer graphene/PMMA stack on Si/Sio2/ Cr markers substrate with water bubbles. Backe the Si/Sio2/ Cr markers/graphene/PMMA for evaporate water bubbles and improve the contact between the stack and the substrate, b) after 2 min c) after $15 \mathrm{~min}$.

After the transfer of PMMA/graphene stack onto another substrate, remove PMMA with NMP, Clean with IPA, and then dry with low pressure ultra high purity. Typically after PMMA removal, put the Si/Sio2/graphene in vacuum sputtering with argon gas at a temperature of $300{ }^{\circ} \mathrm{C}$ for 20 minutes to be used to further improve the graphene/substrate contact and remove residual particles of PMMA. The end of step of transfer, sample clean with acetone, IPA, DI water, and then dry with low pressure ultra high purity N2. Graphene transfer process as shown in Figure 6. 


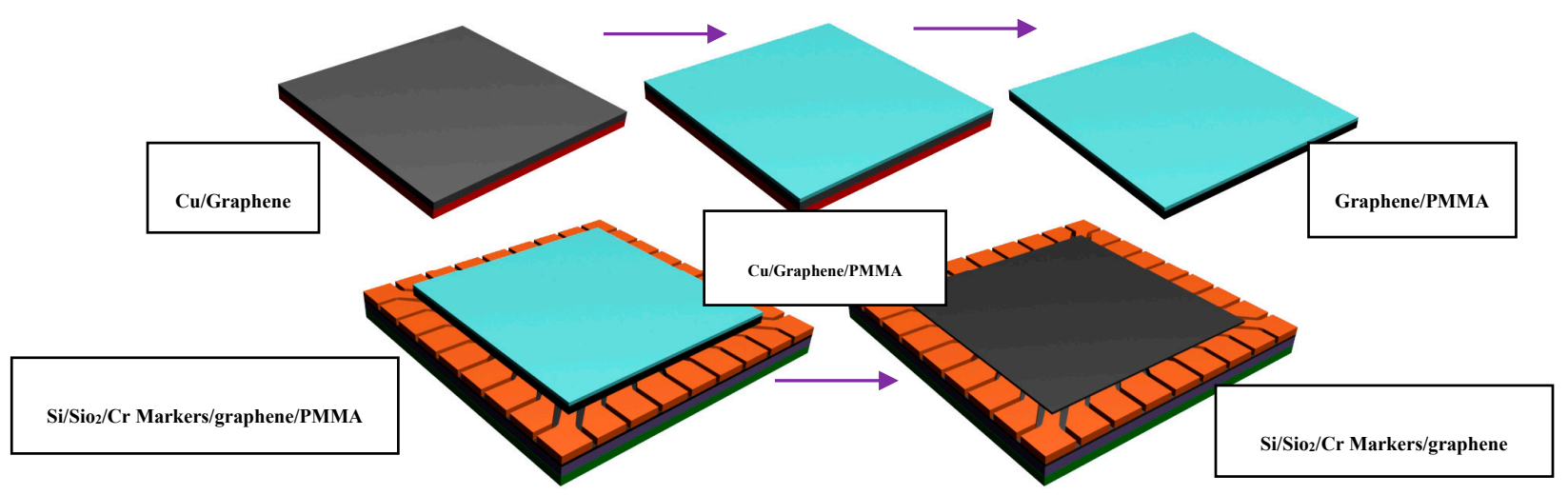

Figure 6. Graphene transfer process.

After graphene has been successfully transferred onto Si/Sio2 substrates, it is ready to be fabricated into electronic devices. The graphene sheet was first patterned into $(60 \times 56) \mu \mathrm{m} 2$ and (30×56) $\mu \mathrm{m} 2$ stripes by using an oxygen plasma. Graphene patterning mask, with rectangles equal to the area between the electrodes (Rectangular $60 \times 56 \mu \mathrm{m} 2 \& 30 \times 56 \mu \mathrm{m} 2$ ) in the markers mask is designed. Graphene patterning mask with the SEM images as shown in Figure 7. The graphene sheet was patterned into (Rectangular $60 \times 56 \mu \mathrm{m} 2 \& 30 \times 56 \mu \mathrm{m} 2$ ) by using an oxygen plasma, for 10 minutes, with power $25 \mathrm{w}, \mathrm{O} 2=25 \mathrm{Sccm}, 350 \mathrm{~m}$ torr pressure in order to removing the graphene sheets without the PMMA coating.
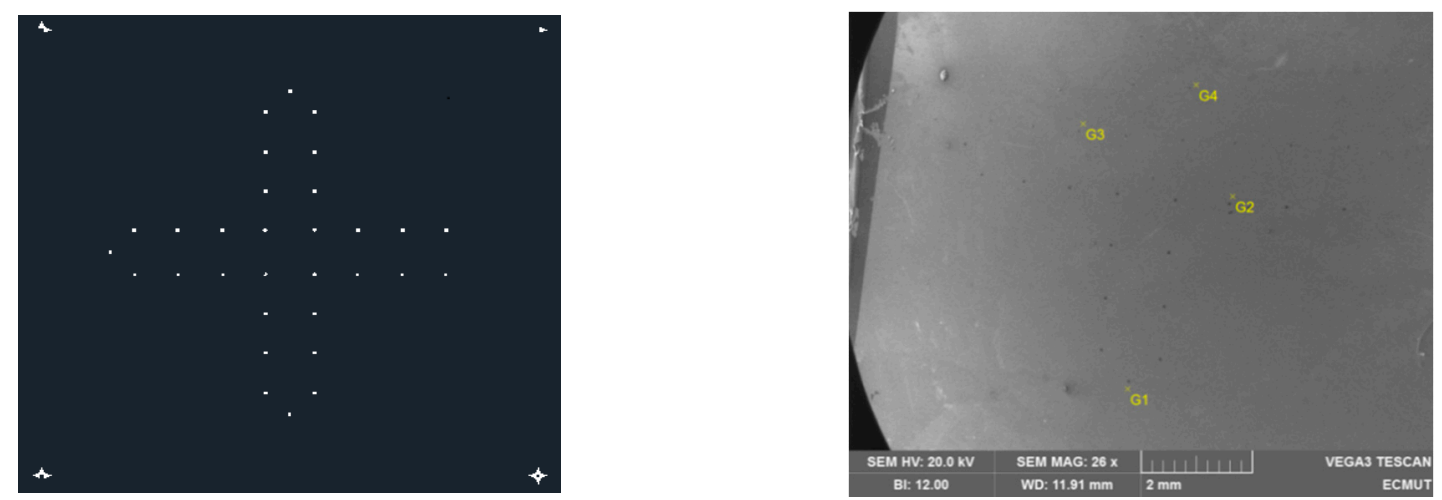

Figure 7. Graphene patterning mask, the SEM images of the graphene mask (Rectangular $60 \times 56 \mu \mathrm{m} 2 \&$ $30 \times 56 \mu \mathrm{m} 2)$, to protect graphene in $\mathrm{O} 2$ plasma process (G1, G2, G3 and G4).

\section{Device Fabrication}

\subsection{Designing mask and deposition of electrodes}

The utilized pattern is designed with markers on the top and down, as Figure 8, by magnifying 2 sections which have 31 transistor samples with different dimensions ( $w=60$ micron \& $\mathrm{L}=6$ micron, $\mathrm{w}=30$ micron \& $\mathrm{L}=6$ micron). To apply drain and source electrodes, (110 $\mathrm{nm} \mathrm{Au}$ on $30 \mathrm{~nm} \mathrm{Cr}$ ) were fabricated by standard photolithography processes based on a widely used photoresist, Shipley 1813. After metal deposition, lift-off was performed at $80^{\circ} \mathrm{C}$ by soaking the sample in Remover 1165 (1-methyl-2-pyrrolidinone) and then thoroughly rinsed with isopropyl alcohol. This process will strip the photoresist from the graphene surface relatively cleanly. 

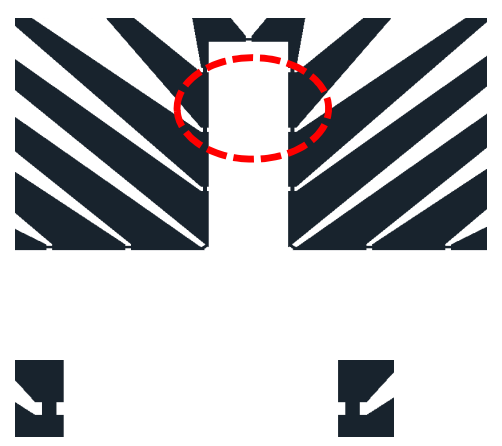

$W=60^{\mu m} \quad L=56^{\mu m}$

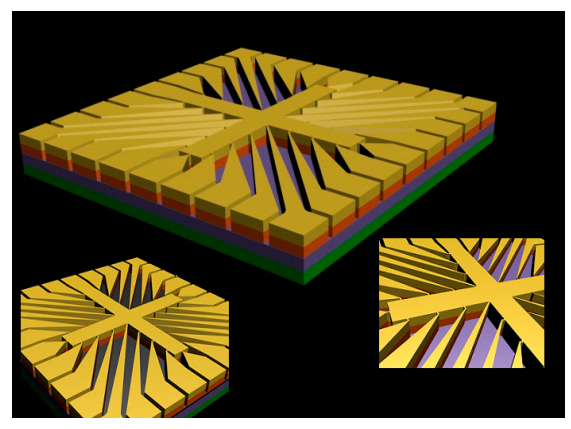

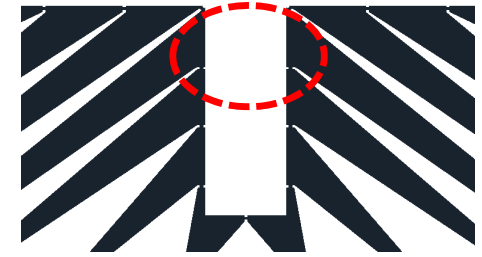

$W=30^{\mu m} \quad L=56^{\mu m}$

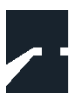

Figure 8. The mask of the source and drain electrodes.

An important feature of lift processes is that the side wall profile of the photoresist must be vertical or with an overhang. This causes a break in the deposited metal film and ensures easy lift-off. After normal lithography the side walls are sloping and in order to get an overhang profile the lithographic process is altered slightly. A toluene (formerly chlorobenzene) soak step is included after the UV exposure but before development. The toluene hardens the top layers of the photo resist making them harder to develop away. In general, the exposure time and the development time need to be changed from the optimal conditions to account for the alteration of the resist properties due to the toluene soak. After the expose sample to UV light, immerse the sample in a beaker containing toluene for 1 minute and do not rinse the sample in DI $\mathrm{H} 2 \mathrm{O}$ after the toluene soak. Then now develop the photoresist and the other standard lithography process can be continued. The size of the whole device is $1.45 \times 1.45 \mathrm{Cm} 2$. To enhance the adhesion between the $\mathrm{Au}$ and the Si wafer, a $30 \mathrm{~nm}$ thick $\mathrm{Cr}$ layer was used. The (SEM) images of the GFETs sensor with $\mathrm{Cr}-\mathrm{Au}$ electrodes are illustrated in Figure 9. Electrical properties of the final devices were measured by using an Agilent 4145B semiconductor parameter analyzer.
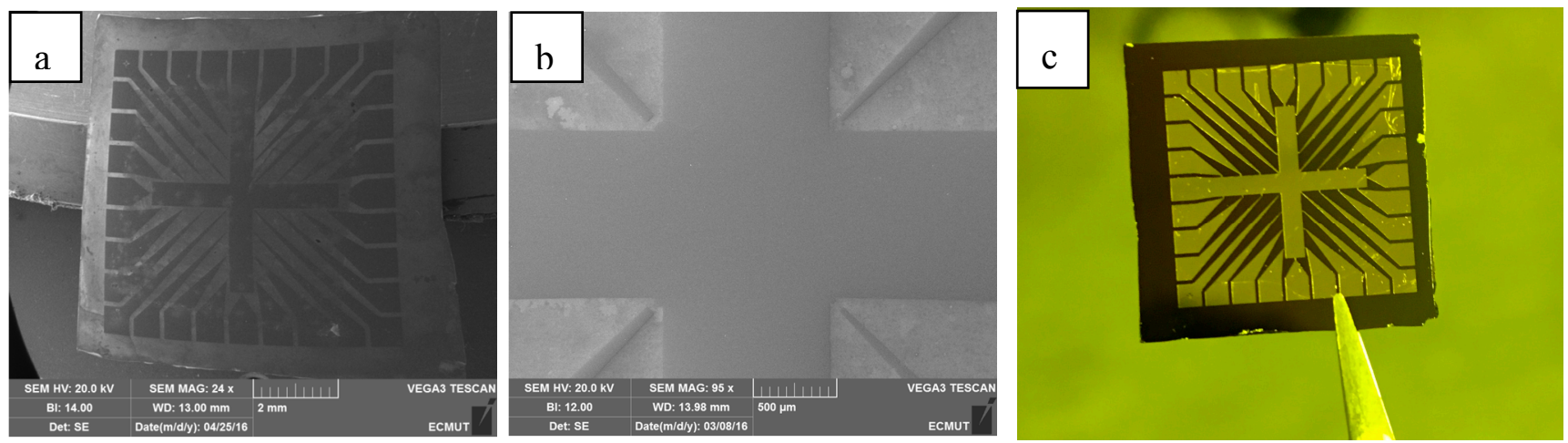

Figure 9. a) The SEM image of GFETs sensor. b) The SEM image of the source and drain electrodes. c) The made GFETs sensor.

\section{Bipolar property of GFETs}

Bandgap tenability is beneficial to transfer graphene characteristic from metal to semi-conductive properties. And current-voltage characteristics of graphene transistors indicate the ambipolarity of graphene. Bipolar property in GFET structure means the passing of current by electrons in a range of gate voltage and by holes in another range of gate voltage [18]. Dirac point means zero concentration of carriers when the field is zero. In an ideal case, the transfer characteristic of GFET should be quasi ballistic. Because graphene is a zero band gap, saturation in drain current chart is not created. Through utilization of bipolar gate voltage, the carriers in graphene transform electrons (holes) from holes (electrons) continuously. Negative gate voltage makes holes conductive in graphene. Otherwise, electrons are dominant in graphene under positive 
gate voltage. So resistance or conductivity of graphene device changes with the variable gate voltage. Leakage current between the channel and gate is a critical factor for device reliability when making graphene gate controlled electronic devices, and more and more researchers are devoted to diminishing the leakage current. The GFET exhibits very small leakage current between the channel and gate due to the channel and the gate dielectric material being separated by an ultrathin blocked graphene connection in the FET device. To investigate the electrical properties of the GFETs device, the output and transfer characteristics of the GFETs sensor were obtained. As shown in Figures 10, 11 the typical Ids - Vds curves were obtained to further examine the electrical characteristics of the GFETs device. The drain-source current decreases with a slight reduction of the gate voltage, indicating that the device reaction is sensitive to the gate voltage. Figure $12 \mathrm{a}$ and $\mathrm{b}$ are typical transfer properties of graphene FETs. The transfer characteristic curve (Ids as a function of back-gate voltage, $\mathrm{Vg}$ ) is smooth with little hysteresis, and the conductivity minimum associated with the Dirac point is near $1 \mathrm{~V}$. Dirac point, which can be identified as the charge neutrality point (CNP). These behaviours resemble the ambpolar field effect observed in graphene. These results indicate that the surface contamination due to the graphene transfer process is very low; therefore, the device performance is repeatable without any annealing treatment (thermal or current) needed. The current between the source and drain electrodes in different devices, related to the shape of the graphene positioning in channel.

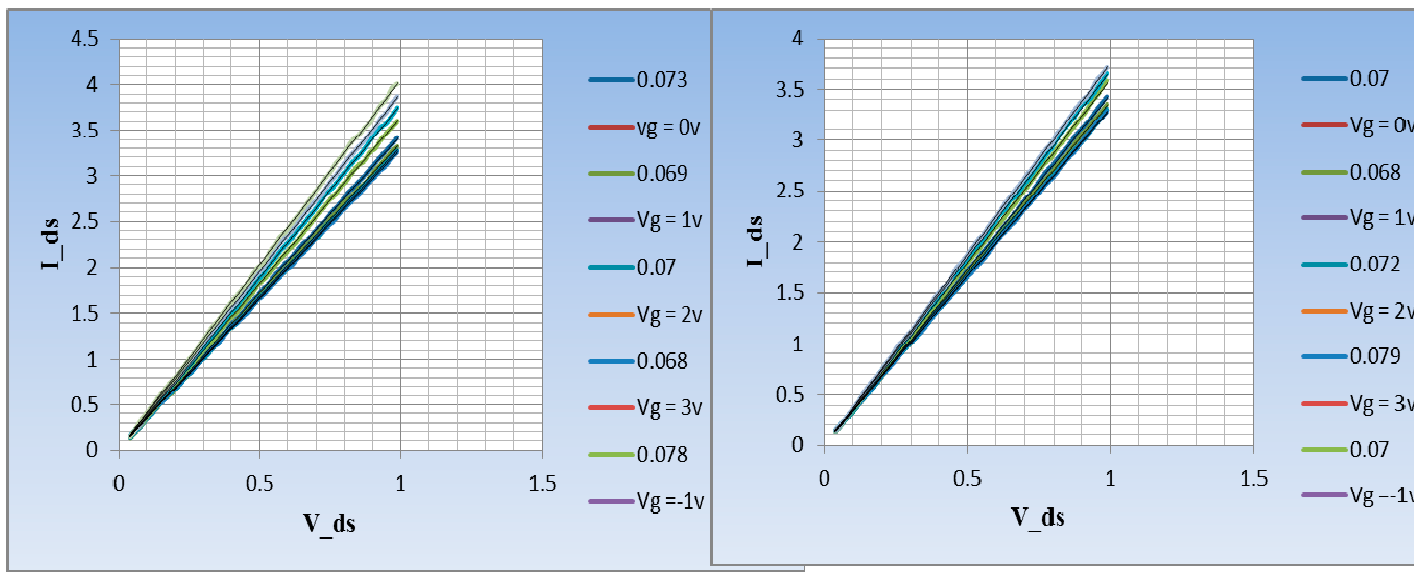

Figure 10. Electrical Properties of GFETs devices. Ids - Vds Output characteristics of the GFETs device with $\mathrm{W} / \mathrm{L}=5$ electrodes. a) The gate voltage $\mathrm{Vg}$ is varied from ( -4 to 3 volt) with an interval of $1 \mathrm{~V}$. b) The gate voltage $\mathrm{Vg}$ is varied from (-3 to 3 volt) with an interval of $1 \mathrm{~V}$. 


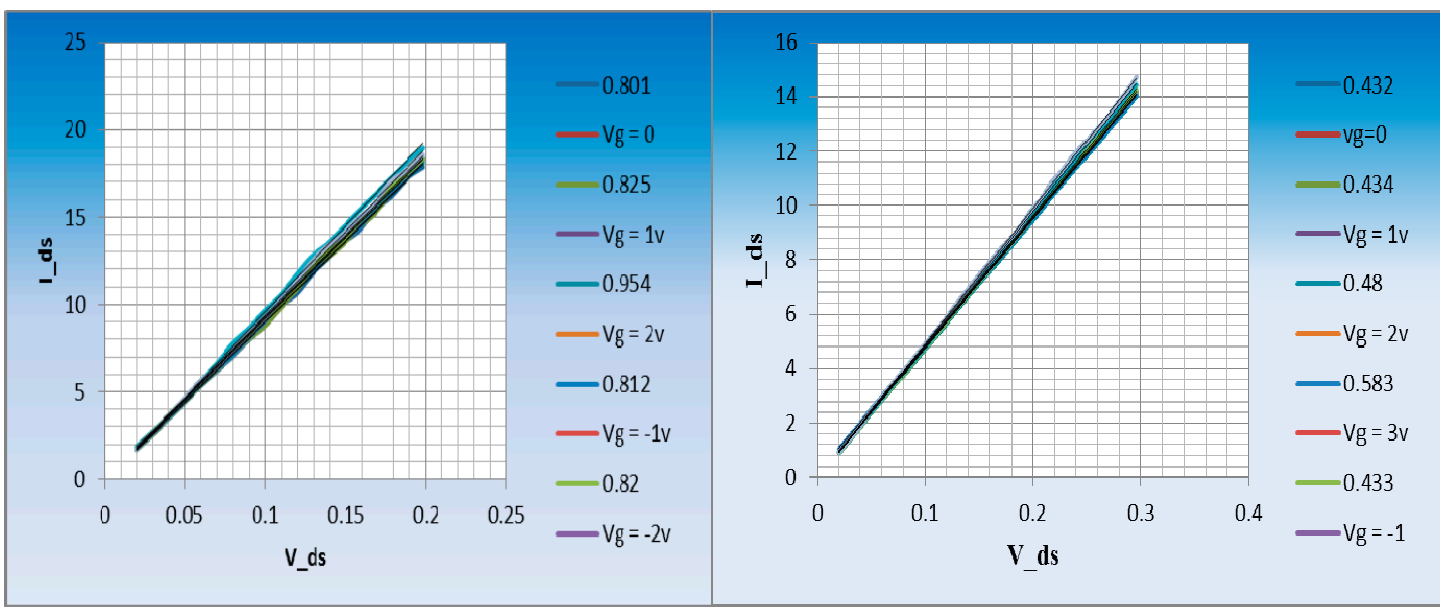

Figure 11. Electrical Properties of GFETs devices .Ids - Vds Output characteristics of the GFETs device with $\mathrm{W} / \mathrm{L}=10$ electrodes. a) The gate voltage $\mathrm{Vg}$ is varied from (-4 to 3 volt) with an interval of $1 \mathrm{~V}$. b) The gate voltage $\mathrm{Vg}$ is varied from (-3 to 3 volt) with an interval of $1 \mathrm{~V}$.

Current measurements between terminals source and drain electrodes with $\mathrm{W} / \mathrm{L}=10$ overlap very well.
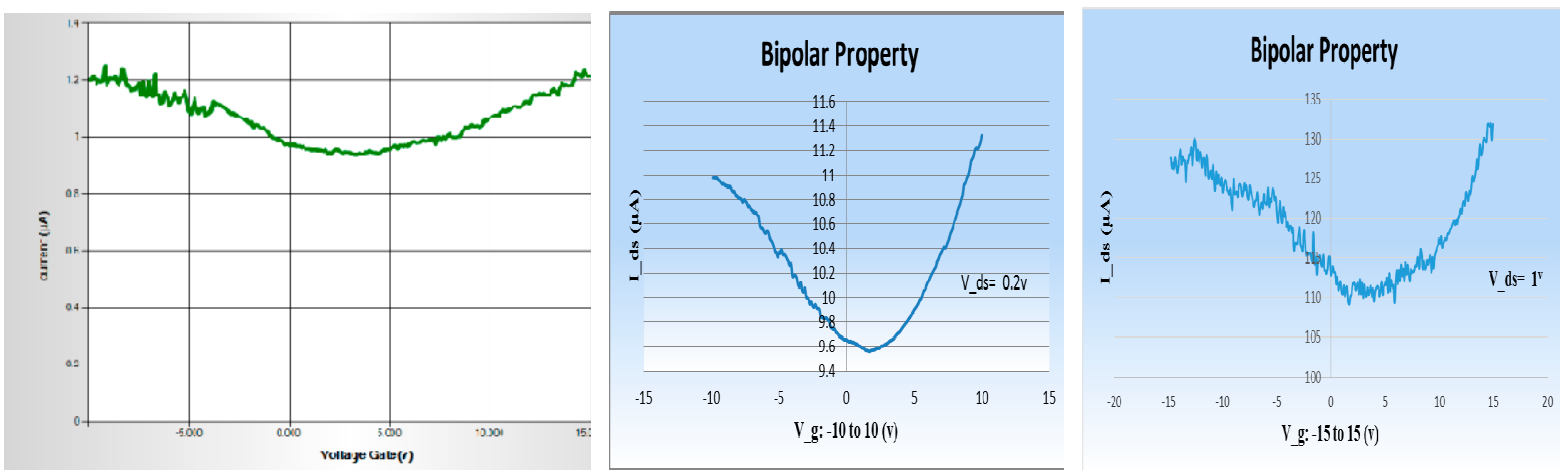

Figure 12. a and b Typical electrical measurement results.

The hysteresis of the Ids - Vds curve between electrodes with $\mathrm{W} / \mathrm{L}=10$ are small. The gate leakage current was in the nA range for all measurements as shown in Figure 13.
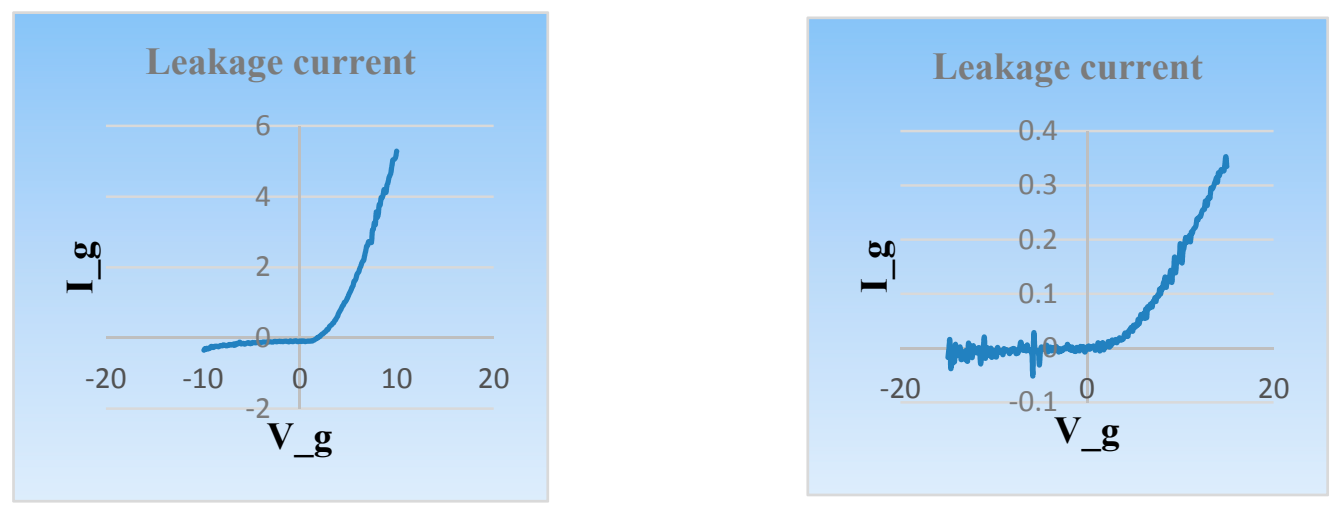

Figure 13. The gate leakage current of tow devices.

To study the uniformity of devices fabricated from graphene films transferred via the RCA clean process, 60 devices were measured and the distribution of the Dirac point is shown in Figure 
14. These results indicate that the graphene transfer is very clean and the graphene quality in the entire device channel is very uniform. The next step is for functionalized GFETs with biological receptors and applies them to the sensor for real-time detection and identification of explosives that are selective [19] for TNT is sensitive done.

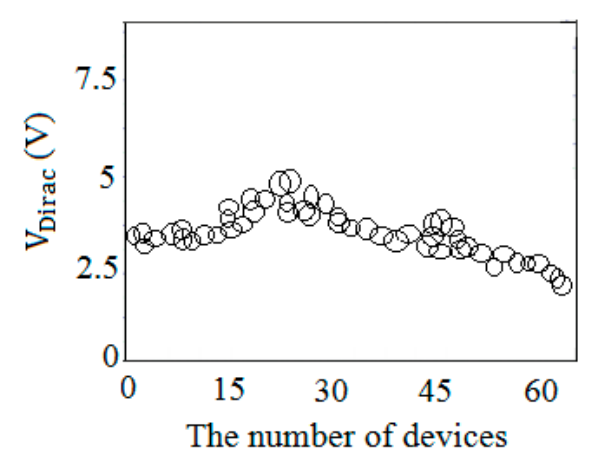

Figure 14. Distribution of Dirac point.

\section{Designing Polydiacetylence receptor}

Several groups have exploited $\pi$-conjugated polymers, particularly polydiacetylenes (PDAs), for chemical sensing applications because of the ability of their absorption spectra to change in response to target binding [20], [21], [22]. PDA amphiphilic polymer composed of a carboxylic head group and an alkyl tail that facilitates its formation into supermolecular assemblies [10]. When these assemblies are exposed to UV irradiation, a conjugated PDA (ene-yne) carbon backbone is formed that exhibits strong absorbance peaks at 640 or $540 \mathrm{~nm}$ resulting in a blue or red color, respectively [23]. The structure of backbone is like Polydiacetylence and absorption spectrum is like Figure 15.
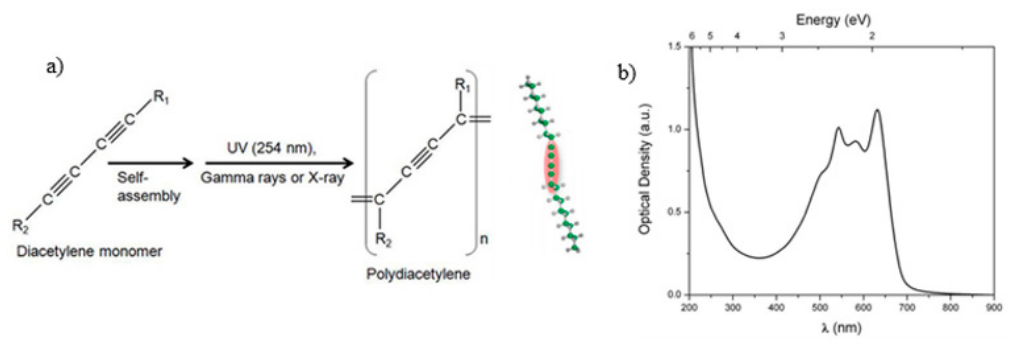

Figure 15. a) Backbone like structure. b) Polydiacetylence of PDA absorption spectrum with peaks in 540 and 640 nanometer.

PDA is used vastly because of sensitivity and capability of changing absorption spectrum as the length of effective joint shortens due to external stimulus [24]. External stimulus, similar to surface or connection disorders, can induct pressure and distortion within the lateral chains of PDA, which results in structural transfer in the backbone [10]. This shift breaks the flatness of the conjugated backbone network and, consequently, absorption in lower wavelengths, and, hence, a red-color transfer increases [10]. Molecule interactions as a result of disorder in surface hydrogen connections, for making enough stress in polymer backbone by affecting the length of disordered polymer is proved. This change in length of effective connection of $\pi$ conjugate backbone can be observed directly through PDA absorption spectrum, so it is ideal for sensing purposes. PDA with 2-4-6 trinitrotoluene receptor, (Trp-His-Trp) mingles with considered proportions (4:96 molar ratio between Trp-His-Trp-PDA and PDA) and is used for functionalizing the Graphene-based sensors . TNT receptor has 12 components amino acid where the first 3 components are considered as an active location [25]. 


\section{Materials and methods}

\subsection{Synthesis of TNT receptor, is connected to the PDA and applies it to the sensor}

We characterized the multivalent binding interaction between TNT target molecules and the peptide TNT receptor (Trp-His-Trp). This indicates that one of the major driving forces of the (Trp-His-Trp). TNT complex formation is $\pi-\pi$ interactions between tryptophan and TNT's aromatic ring structure [26]. We believe that these $\pi-\pi$ interactions of the (Trp-His-Trp).TNT complex induce the electronic band structure perturbation of Trp-His-Trp-PDA upon binding with TNT target molecules in a multivalent binding manner [26]. The receptor of TNT explosive is in the form of three amino acids (Trp-His-Trp) which is synthesized. TNT biological receptors (Trp-His-Trp) are shown in Figures 16 a \& b.
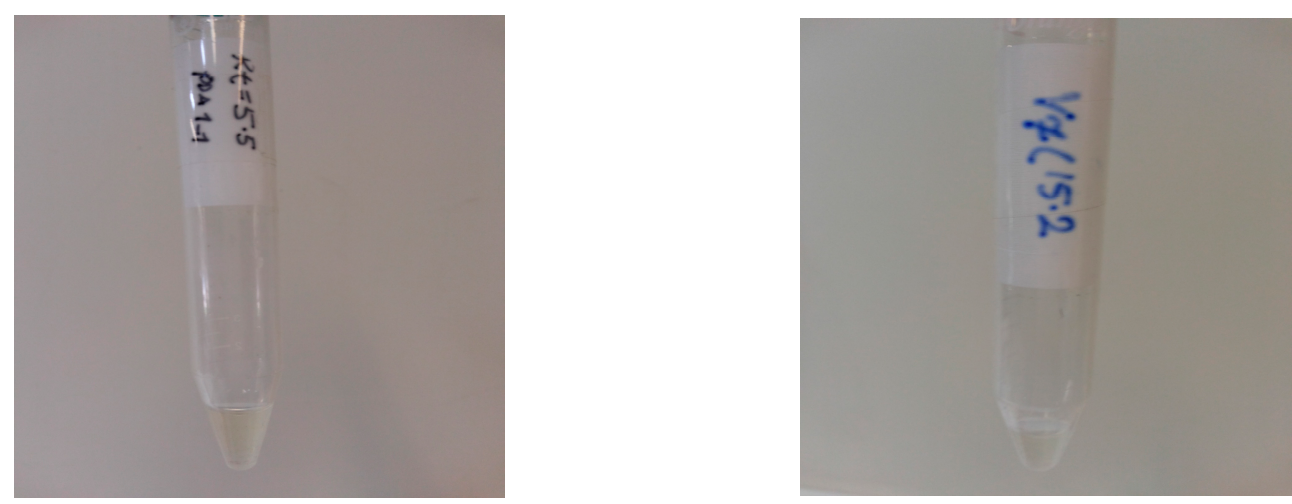

Figure 16. TNT biological receptor.

\subsection{Controlling the Surface Composition and Density in Supramolecular Assemblies}

PDA used in the design was purchased from Sigma Corporation. To produce PDA-peptide conjugates, standard solid-phase peptide synthesis was carried out using FMOC chemistry. The receptor of target molecules is in the form of three amino acids (Trp-His-Trp) which is synthesized and is connected to PDA (5 samples). PDA and TNT-receptor/PDA were suspended in water. Solutions were sonicated for $45 \mathrm{~min}$ and heated at $90^{\circ} \mathrm{C}$ to melt any existing supermolecular structure. To allow self-assembly into vesicle structures, the solution was allowed to cool to room temperature then incubated at $5^{\circ} \mathrm{C}$ for $24 \mathrm{~h}$. According to the outcomes and synthesizing peptide samples we shall choose the best among the samples and apply it to the transistor. Block diagram of GFETs sensor, with graphene sheet as transistor channel, biological receptors explosive TNT with PDA is shown in Figure 17a. The schematic diagram shows TNT explosives sensors based on Trp-His-Trp -PDA (TNT-binding peptide conjugated with polydiacetylene polymer) functionalized GFETs. After formation of TNT receptor-PDA/PDA vesicles in H2o, we applied the vesicles to a sensor, which resulted in rupture, fusion, and UV polymerization of the Trp-His-Trp-PDA/PDA membranes on the GFETs. The molecules and GFETs sensor are not drawn to scale.

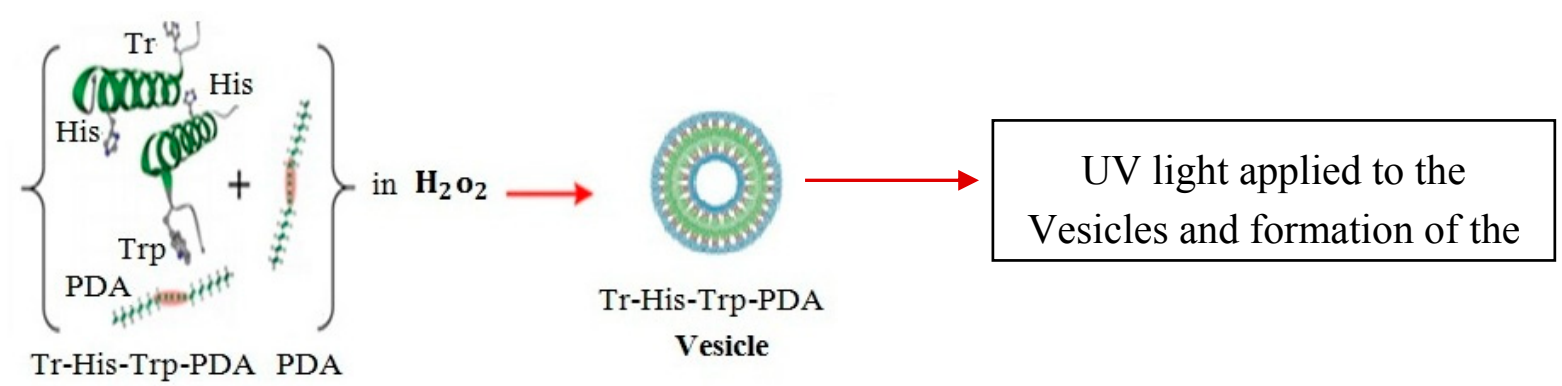



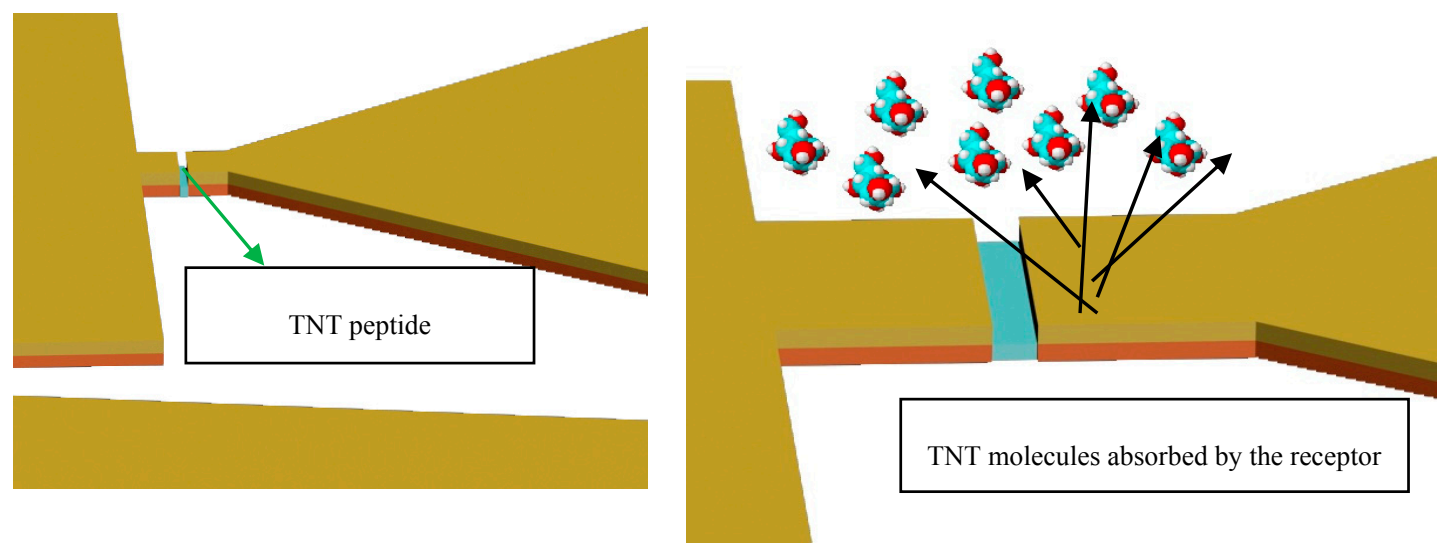

Figure 17. Block diagram of GFETs sensor, with graphene sheet as a transistor channel and biological receptors with PDA to detect explosive TNT.

\section{RESULTS}

The device was annealed at $300^{\circ} \mathrm{C}$ for $15 \mathrm{~min}$ at an ambient environment to improve the contact between graphene and electrodes. The sensitivity of the GFET biosensor into target molecules was investigated by various concentrations of TNT with the PDA/ TNT-receptor that functionalized GFET device. The amphiphilic nature of PDA monomers facilitates its formation into supramolecular assemblies such as vesicles and membranes. Finally, the target TNT are applied onto the device and then a silver wire is used as the gate to realize a gated GFET for electrical measurements. In principle, the $\pi-\pi$ interactions of the (Trp-His-Trp) \& TNT causes n-doping of the devices due to graphene. The source- drain current and back-gate voltage (Ids - Vg) curves of the pristine graphene FET measured in air Figure 12 and show bipolar transistor effect, and the minimal conduction corresponds to the Dirac point (Vdirac). When $\mathrm{Vg}<$ Vdirac, it operates as a p-channel FET, and for Vg > Vdirac, the device operate as an n-channel FET. In our transport measurement, the graphene annealed in TNT after irradiation shows distinguishing transport features in air Figure 18 compared with the pristine graphene FET and the FET. Figure $18 \mathrm{~d}$ shows, when the GFET sensor coated with PDA without receptor, the electrical current of the sensor exhibited almost No changes to various concentrations of TNT molecules. 

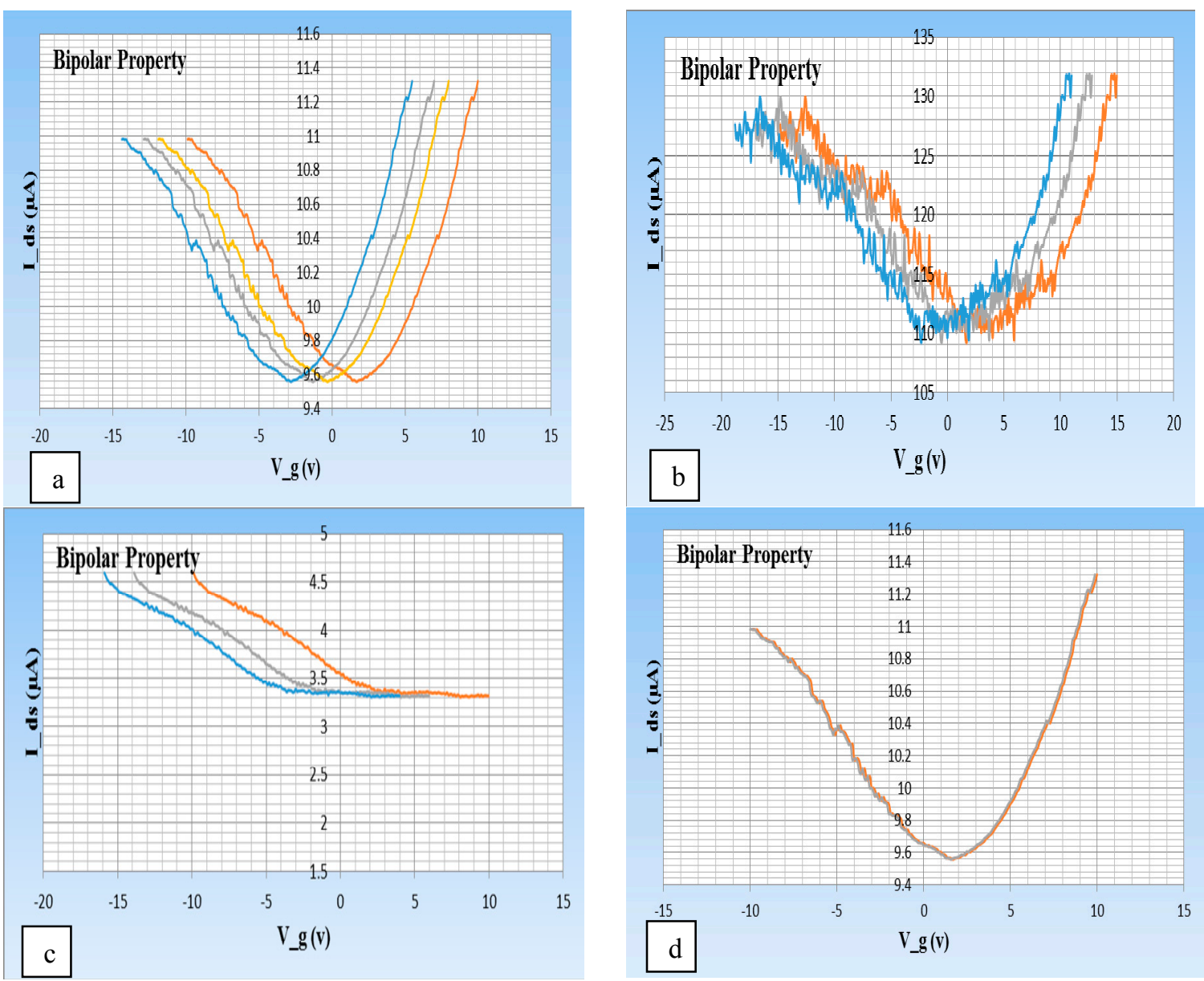

Figure 18. The Ids - Vg curves of graphene annealed in TNT after irradiation in GFETs. a) curves are measured at $\mathrm{Vsd}=0.2 \mathrm{~V}$. b) $\mathrm{Vsd}=1 \mathrm{~V}$. c) $\mathrm{Vsd}=1 \mathrm{~V}$. d) GFET sensor coated with PDA without receptor and No changes in bipolar property curve.

We could see that the biploar property of the GFETs shifted toward the left with increased concentration of the TNT, and this result is in good agreement with that described by other reports [10].

\section{Conclusions}

In summary, we have studied clean methods for transferring graphene from $\mathrm{Cu}$ substrates to target substrates and fabricated the FET biosensors based on graphene with biological receptor for specific detection of TNT explosive and graphene sheet is controllably placed between the electrodes. The graphene/wafer-substrate interface resulting from this clean transfer process is greatly improved. Lab results shows that the number of cracks in transferred graphene films can be reduced by controlling the hydrophilicity of the target substrate and baking. Graphene as the conducting material shows the obvious ambipolar characteristics at a small range of gate voltage on the back-gated FET devices under ambient conditions, and the devices are highly responsive to the low gate voltage. In order to functionalize the made GFETs as a sensor with ability of recognizing the explosive TNT, the biological receptors were designed and synthesized. We have developed a rapid and selective TNT sensor based on FET with nanomaterials with good sensitivity and selectivity through the combination of GFETs and PDA-based lipid membranes coupled with 
biologically inspired TNT peptide receptors to significantly improve selectivity in a Nano electronic device sensor .In our system, a sensitive GFET interfaced with PDA was exploited to transduce the binding activities between the target TNT and its TNT selective binding peptide receptor. Conjugated PDA-based lipid-like polymers provided a stable coating platform to interconnect the selective TNT receptor and sensitive FET devices. Apply various types of explosives to the sensor, (Trp-His-Trp/PDA functionalized Sensor) does not show that any response to non-target molecules and only in the presence target molecules of TNT reacts. Sensor recognition accuracy is at an acceptable level .Design and manufacture of explosives detector sensors based on GFETs that capable of responding to several different types of explosives in real time, is the next thing the research group will publish in this journal.

\section{References}

1. Kong, J.; Franklin, N.R.; Zhou, C.; Chapline, M.G.; Peng, S.; Cho, K.; Dai, H. Nanotube molecular wires as chemical sensors. Science 2000, 287, 622-625.

2. Cai, B.; Wang, S.; Huang, L.; Ning, Y.; Zhang, Z.; Zhang, G.-J. Ultrasensitive label-free detection of pna-DNA hybridization by reduced graphene oxide field-effect transistor biosensor. ACS nano 2014, $8,2632-2638$.

3. He, R.X.; Lin, P.; Liu, Z.K.; Zhu, H.W.; Zhao, X.Z.; Chan, H.L.; Yan, F. Solution-gated graphene field effect transistors integrated in microfluidic systems and used for flow velocity detection. Nano letters 2012, 12, 1404-1409.

4. Zhan, B.; Li, C.; Yang, J.; Jenkins, G.; Huang, W.; Dong, X. Graphene field-effect transistor and its application for electronic sensing. Small 2014, 10, 4042-4065.

5. Anderson, G.P.; Moreira, S.C.; Charles, P.T.; Medintz, I.L.; Goldman, E.R.; Zeinali, M.; Taitt, C.R. Tnt detection using multiplexed liquid array displacement immunoassays. Analytical chemistry 2006, 78, 2279-2285.

6. Goldman, E.R.; Medintz, I.L.; Whitley, J.L.; Hayhurst, A.; Clapp, A.R.; Uyeda, H.T.; Deschamps, J.R.; Lassman, M.E.; Mattoussi, H. A hybrid quantum dot- antibody fragment fluorescence resonance energy transfer-based tnt sensor. Journal of the American Chemical Society 2005, 127, 6744-6751.

7. Bogue, R. Detecting explosives and chemical weapons: A review of recent developments. Sensor Review 2015, 35, 237-243.

8. Novoselov, K.S.; Geim, A.K.; Morozov, S.V.; Jiang, D.; Zhang, Y.; Dubonos, S.V.; Grigorieva, I.V.; Firsov, A.A. Electric field effect in atomically thin carbon films. science 2004, 306, 666-669.

9. Lemme, M.C.; Echtermeyer, T.J.; Baus, M.; Kurz, H. A graphene field-effect device. IEEE Electron Device Letters 2007, 28, 282-284.

10. Masoumi, S.; Masoumi, S.; Hajghassem, H.; Hajghassem, H.; Erfanian, A.; Erfanian, A.; Molaei Rad, A.; Molaei Rad, A. Design and manufacture of tnt explosives detector sensors based on cntfet. Sensor Review 2016, 36, 414-420.

11. Reina, A.; Son, H.; Jiao, L.; Fan, B.; Dresselhaus, M.S.; Liu, Z.; Kong, J. Transferring and identification of single-and few-layer graphene on arbitrary substrates. The Journal of Physical Chemistry C 2008, 112, 17741-17744.

12. Koratkar, N.A. Graphene in composite materials: Synthesis, characterization and applications. DEStech Publications, Inc: 2013.

13. Liang, X.; Sperling, B.A.; Calizo, I.; Cheng, G.; Hacker, C.A.; Zhang, Q.; Obeng, Y.; Yan, K.; Peng, H.; Li, Q. Toward clean and crackless transfer of graphene. ACS nano 2011, 5, 9144-9153. 
14. Li, X.; Cai, W.; An, J.; Kim, S.; Nah, J.; Yang, D.; Piner, R.; Velamakanni, A.; Jung, I.; Tutuc, E. Large-area synthesis of high-quality and uniform graphene films on copper foils. Science 2009, 324, 1312-1314.

15. Jo, G.; Choe, M.; Cho, C.-Y.; Kim, J.H.; Park, W.; Lee, S.; Hong, W.-K.; Kim, T.-W.; Park, S.-J.; Hong, B.H. Large-scale patterned multi-layer graphene films as transparent conducting electrodes for gan light-emitting diodes. Nanotechnology 2010, 21, 175201.

16. Nagashio, K.; Yamashita, T.; Fujita, J.; Nishimura, T.; Kita, K.; Toriumi, A. In Impacts of graphene/sio 2 interaction on fet mobility and raman spectra in mechanically exfoliated graphene films, Electron Devices Meeting (IEDM), 2010 IEEE International, 2010; IEEE: pp 23.24. 21-23.24. 24.

17. Nagashio, K.; Yamashita, T.; Nishimura, T.; Kita, K.; Toriumi, A. Electrical transport properties of graphene on sio2 with specific surface structures. Journal of Applied Physics 2011, 110, 024513.

18. Kim, W.; Javey, A.; Vermesh, O.; Wang, Q.; Li, Y.; Dai, H. Hysteresis caused by water molecules in carbon nanotube field-effect transistors. Nano Letters 2003, 3, 193-198.

19. Chen, Y.; Chen, Z.; He, Y.; Lin, H.; Sheng, P.; Liu, C.; Luo, S.; Cai, Q. L-cysteine-capped cdte qd-based sensor for simple and selective detection of trinitrotoluene. Nanotechnology 2010, 21, 125502.

20. Biesalski, M.; Tu, R.; Tirrell, M.V. Polymerized vesicles containing molecular recognition sites. Langmuir 2005, 21, 5663-5666.

21. Kohler, A.; Hoffmann, S.T.; Bässler, H. An order-disorder transition in the conjugated polymer meh-ppv. Journal of the American Chemical Society 2012, 134, 11594-11601.

22. Ahn, D.J.; Kim, J.-M. Fluorogenic polydiacetylene supramolecules: Immobilization, micropatterning, and application to label-free chemosensors. Accounts of chemical research 2008, 41, 805-816.

23. Carpick, R.W.; Sasaki, D.Y.; Marcus, M.S.; Eriksson, M.; Burns, A.R. Polydiacetylene films: A review of recent investigations into chromogenic transitions and nanomechanical properties. Journal of Physics: condensed matter 2004, 16, R679.

24. Cerruti, M.; Jaworski, J.; Raorane, D.; Zueger, C.; Varadarajan, J.; Carraro, C.; Lee, S.-W.; Maboudian, R.; Majumdar, A. Polymer-oligopeptide composite coating for selective detection of explosives in water. Analytical chemistry 2009, 81, 4192-4199.

25. Jaworski, J.W.; Raorane, D.; Huh, J.H.; Majumdar, A.; Lee, S.-W. Evolutionary screening of biomimetic coatings for selective detection of explosives. Langmuir 2008, 24, 4938-4943.

26. Jaworski, J.; Yokoyama, K.; Zueger, C.; Chung, W.-J.; Lee, S.-W.; Majumdar, A. Polydiacetylene incorporated with peptide receptors for the detection of trinitrotoluene explosives. Langmuir 2011, 27, 3180-3187. 